\title{
Estructura del Bienestar Subjetivo Mediante Análisis Bifactor: ¿Unidimensional o Multidimensional?
}

\author{
Lucas Lapuente \\ Secretaría de Investigación, Universidad Siglo 21, Córdoba, Argentina \\ Sergio Dominguez-Lara \\ Instituto de Investigación de Psicología, Universidad de San Martín de Porres, Lima, Perú \\ Pablo Ezequiel Flores-Kanter, Leonardo Adrián Medrano ${ }^{1}$ \\ Secretaría de Investigación, Universidad Siglo 21, Córdoba, Argentina
}

\section{RESUMEN}

En la presente investigación se analizó la estructura del bienestar subjetivo (BS) propuesto por Diener (1985) aplicando un modelo bifactor. Participaron 281 estudiantes cordobeses (61.2\% mujeres; $\left.M_{\text {edad }}=21.7\right)$ que fueron evaluados con la Satisfaction with Life Scale y la Positive and Negative Affect Schedule, siendo la primera una medida de satisfacción con la vida (SV) y la segunda, de afecto positivo (AP) y negativo (AN). Fue realizado el análisis factorial confirmatorio con el fin de evaluar dos modelos: oblicuo (AP, AN, y SV) y bifactor, con un factor general (FG) que representan el BS, y tres específicos. El modelo bifactor tuvo mejor ajuste, aunque los resultados obtenidos (e.g., $\omega_{h}$ ) indican que el FG sólo explica el 15,9\% de la variabilidad de las puntuaciones. Por eso, el modelo propuesto por Diener (1985) tiene mayor respaldo, demostrando evidencia sobre la multidimensionalidad del BS al diferenciar aspectos afectivos y cognitivos.

Palabras clave: bienestar subjetivo; satisfacción con la vida; afecto; modelo bifactor.

\section{RESUMO - Estrutura do Bem-Estar Subjetivo Mediante Análise Bifator: Unidimensional ou Multidimensional?}

Na presente pesquisa, foi analisada a estrutura do Bem-Estar Subjetivo (BS) proposto por Diener (1985), aplicando um modelo bifatorial. Participaram 281 estudantes cordobeses $\left(61,2 \%\right.$ mulheres, $\left.M_{i d a}=21,7\right)$, que foram avaliadas com a Satisfaction with Life Scale e a Positive and Negative Affect Schedule, sendo a primeira a medida de satisfação com a vida (SV) e a segunda, de afeto positivo (AP) e negativo (AN). Foi executada a análise fatorial confirmatória para avaliar dois modelos: oblíquo (AP, AN, y SV) e bi-fatorial, com um fator geral (FG) que representa BS, e três específicos. O modelo bi-fatorial teve melhor ajuste, embora os resultados obtidos (por exemplo, $\omega_{h}$ ) indicaram que o FG só explica o $15,9 \%$ da variabilidade dos scores. Por isso, o modelo proposto por Diener (1985) tem maior respaldo, o que fornece evidência sobre a multidimensionalidade do BS ao diferenciar afetivos e cognitivos.

Palavras-chave: bem-estar subjetivo; satisfação com a vida; afeto; modelo bifactor.

\section{ABSTRACT - Subjective Well-Being, Unidimensional or Multidimensional? Bi-Factor Analysis in a Sample of College Students}

In the present study the subjective well-being structure (WB) proposed by Diener (1985) was analyzed through application of a bifactor model. A total of 281 students from Córdoba, Argentina, 61.2\% women, $M_{\text {age }}=21.7$, participated in the study, and were evaluated with Satisfaction with Life Scale (LS), and the Positive and Negative Affect Schedule (PA and NA, respectively). Confirmatory factor analysis was performed to evaluate two models: oblique (PA, NA and LS) and bi-factor with a general factor (GF) representing WB, and three specific ones. The bi-factor model had a better fit, although results obtained (e.g., $\left.\omega_{h}\right)$ indicate that GF only accounts for $15.9 \%$ of the variability of scores. For this reason, the model proposed by Diener (1985) has more support, which shows evidence on the multidimensionality of WB by differentiating affective and cognitive.

Keywords: subjective well-being; satisfaction with life; affection; bi-factor model.

El estudio el Bienestar Subjetivo (BS) ha crecido exponencialmente en la última década (Busseri, 2015; Luhmann, 2017). Sin embargo, todavía persisten controversias en relación a su definición conceptual y operacional. En algunos casos aparece como sinónimo de salud mental, resiliencia o felicidad, no quedando en claro si existe un solapamiento entre dichos conceptos o simplemente una confusión respecto a su uso (Olivier, Navarro-Guzmán, Menacho-Giménez, López-Sinoga \& García-Sedeño, 2016). En la literatura especializada aún se discute sobre la diferenciación entre el BS y el bienestar psicológico, aunque muchos autores la 
desestiman por considerarlos redundantes (GarcíaViniegras \& González-Benítez, 2000).

Probablemente el modelo más popular sobre el BS sea el propuesto por Diener y su equipo, quienes lo definen como "la evaluación cognitiva y afectiva de una persona sobre su propia vida" (Diener, Lucas, \& Oishi, 2002; p. 63). Este planteamiento diferencia tres componentes: la satisfacción con la vida (SV), los afectos negativos (AN) y los afectos positivos (AP). Para algunos autores esta estructura tripartita ha sido raramente cuestionada y ha sido adoptada como una premisa fundamental en los estudios sobre el bienestar subjetivo (Busseri, 2015; Oliveira, Nunes, Legal, \& Noronha, 2016).

A pesar de los problemas conceptuales para definir el concepto del BS, son pocos los trabajos que se han encargado de someter a prueba empírica la estructura tripartita propuesta por este modelo. Aquellos que lo han hecho se basaron en dos tipos de modelos de análisis: un modelo trifactorial con los factores SV, AP y AN correlacionados; y por el otro lado, un modelo de análisis con un factor de segundo orden que explica la correlación entre los factores mencionados (Busseri \& Sadava, 2011). Sin embargo, ambos modelos presentan limitaciones para explicar el modelo del BS. Por un lado, el modelo trifactorial no logra explicar la correlación entre dimensiones (que podría entenderse a su vez como varianza compartida); y por el otro lado, el modelo con factor de segundo orden no logra evaluar simultáneamente la influencia del factor de segundo orden y de primer orden sobre los indicadores observables, es decir, los ítems (Canivez, 2016).

Recientemente, Jovanovic (2015) ha puesto a prueba el modelo propuesto por Diener, al plantear que el BS es mejor explicado por un modelo jerárquico directo o bifactor (Canivez, 2016; Reise, 2012), es decir, tres factores ortogonales cuya variabilidad se explica mejor por la presencia de un factor general (FG). Para corroborar esta hipótesis, el autor comparó los índices de ajuste del modelo bifactor, con los índices del modelo propuesto por Diener (tres factores oblicuos). Sin embargo, al realizar esos análisis, el autor no utilizó los índices complementarios que podrían dar información sustancial sobre el valor explicativo de los factores analizados (específicos y FG) sobre los ítems. Asimismo, un metaanálisis reciente sobre las relaciones entre los componentes del BS indica que estos poseen varianza relevante independiente del FG (Busseri, 2018).

Dadas las controversias que aún existe acerca de la estructura factorial del BS, y en función de la escasez de evidencia en el contexto argentino, el objetivo del presente trabajo fue replicar los estudios hechos por Jovanovic (2015), es decir, analizar la estructura del BS, pero añadiendo los estadísticos complementarios para analizar el peso explicativo de cada uno de los factores.

\section{El modelamiento bifactor del bienestar subjetivo}

El modelamiento bifactor ha sido utilizado para representar empíricamente modelos teóricos y de medición bajo el supuesto de que las relaciones observadas entre diferentes dimensiones dentro de modelos oblicuos se explican mejor por la presencia un FG (Reise, 2012), sobre todo si estas correlaciones son elevadas. En este caso, se plantea que las relaciones teóricas halladas entre el AP, AN, y SV poseen un FG común: el BS.

Por lo general, se evalúa la fortaleza del modelo bifactor sobre otros modelos competidores considerando los índices de ajuste obtenidos a partir del análisis factorial confirmatorio. Sin embargo, no es recomendable basarse únicamente en los índices de ajuste (Dominguez-Lara, 2016a), ya se deja de lado otros estadísticos que brindarían mayor información sobre la influencia del FG sobre los ítems, en comparación con los factores específicos (Rodriguez, Reise, \& Haviland, 2016). Por ejemplo, toda la variabilidad explicada por el modelo podría dividirse entre varianza verdadera y varianza del error, y en este sentido, la varianza verdadera puede ser cuantificada por medio del omega jerárquico general $\left(\omega_{h}\right.$; Zinbarg, Yovel, Revelle, $\&$ McDonald, 2006) y por subescalas $\left(\omega_{h}\right)$, que brindan información sobre cuanta varianza confiable mantiene cada factor específico cuando se controla la presencia del FG.

No obstante, los $\omega_{h}$, general y específico, no diferencian entre la varianza total relacionada con el FG y aquella que se explica mejor por los factores específicos, y considerando que el objetivo es brindar evidencias a favor del FG, ese indicador por sí mismo no es concluyente. Respecto a ello, el ECV (Explained Common Variance; Sijtsma, 2009) se encarga de cuantificar el porcentaje de la varianza verdadera total que se debe al FG (Reise, Scheines, Widaman, \& Haviland, 2013), y el PUC (Percentage of Uncontaminated Correlations; Reise et al., 2013) informa el porcentaje de correlaciones no contaminadas por la multidimensionalidad y, por ende, mejor explicadas por la presencia del FG. La utilidad del PUC radica principalmente en que ayuda a moderar la interpretación del ECV. Es decir, si su valor supera largamente al ECV, es menos probable sustentar la unidimensionalidad de los resultados (Rodriguez et al., 2016). Esto indica que un $\omega_{h}$ moderado o elevado no garantiza la fortaleza del FG, ya que debe existir un ECV elevado (y, de forma deseable, PUC elevado). A su vez, cada uno de los ítems se vería influido simultáneamente por un factor específico y el FG, y el ECV-I (Stucky, Thissen, \& Edelen, 2013) ayudaría a cuantificar cuánta varianza verdadera del ítem es explicada por el FG y el factor específico.

Como puede apreciarse, la valoración de modelos bifactor, lo que a su vez se extiende a los modelos teóricos que pretenden validar, no puede llevarse a cabo solo analizando los índices de ajuste y el $\omega_{h}$ porque estos son una primera aproximación. Por ello, es necesario realizar un análisis exhaustivo de las opciones analíticas que proveen los modelos bifactor, dado que frecuentemente los índices de ajuste de estos modelos superan a los otros competidores, como los modelos oblicuos (ver Gignac, 2016; Morgan, Hodge, Wells, \& Watkins, 2015), dando 
la impresión que son los que poseen un mayor respaldo empírico, cuando en realidad podría ser distinto.

\section{Método}

\section{Participantes}

Para la realización del presente estudio se contó con la participación de 281 estudiantes universitarios de la ciudad de Córdoba (61.2\% mujeres), con edades comprendidas entre los 17 y los 46 años $\left(M_{\text {edad }}=21.7\right.$; $\left.D E_{\text {edad }}=3.21\right)$. La muestra fue seleccionada por medio de un muestreo no probabilístico accidental. Para lograr una muestra heterogénea, se incluyeron participantes de distintas carreras (36 carreras distintas) y de seis universidades. Todos los participantes accedieron voluntariamente a contestar los cuestionarios, asimismo, se garantizó el anonimato y la confidencialidad de las respuestas.

\section{Instrumentos}

Satisfaction with Life Scale (SWLS; Diener et al.,1985) es un instrumento que consta de cinco items que evalúan el componente cognitivo del bienestar subjetivo. Los ítems son respondidos en una escala tipo Likert con siete opciones de respuesta, que van desde muy en desacuerdo hasta muy de acuerdo. Fue utilizada la versión adaptada al contexto cordobés del SWLS, cuyos resultados mostraron que la estructura monofactorial explicaba el $52 \%$ de la varianza total y una consistencia interna de $\alpha=.756$ (Moyano, Martínez Tais, \& Muñoz, 2013).

Positive and Negative Affect Schedule (PANAS; Watson, Clark \& Tellegn, 1988) es un instrumento que consta de 20 ítems que involucra emociones positivas y negativas. El encuestado debe responder en qué medida experimenta cada una de las emociones descriptas mediante una escala de tipo Likert con cinco opciones de respuesta, siendo muy poco o nada y siempre o casi siempre las opciones extremas. Fue utilizada la versión adaptada al contexto cordobés del PANAS (Medrano, Flores-Kanter, Trógolo, Curarello, \& González, 2015). Los resultados de la adaptación mostraron que la estructura de dos factores consistentes $\left(\alpha_{\mathrm{AP}}=.82 ; \alpha_{\mathrm{AN}}=.83\right)$.

\section{Procedimiento}

En un primer momento se administraron las escalas, de manera conjunta y presencial en las diferentes universidades que formaron parte de la muestra. Todos los participantes accedieron voluntariamente a contestar los cuestionarios, asimismo, se garantizó el anonimato y la confidencialidad de las respuestas. Cabe mencionar que el procedimiento aplicado en la presente investigación fue aprobada por el comité de ética de la Universidad Siglo 21.

\section{Análisis de datos}

Luego de las aplicaciones, los datos fueron tabulados y los ítems fueron analizados descriptivamente, tanto de las medidas de tendencia central y dispersión, como asimetría y curtosis, incluyendo el índice estandarizado de asimetría (SSI; Malgady, 2007).

Fue ejecutado un análisis factorial confirmatorio (AFC) utilizando el programa EQS 6.2 (Bentler \& Wu, 2012) bajo las siguientes condiciones: método de máxima verosimilitud robusto, matrices policóricas bajo el método de Lee, Poon y Bentler (1995) debido a que los ítems son medidas ordinales (Dominguez-Lara, 2014), y especificando la varianza de cada factor a la unidad con el objetivo de obtener coeficientes estandarizados.

Fueron evaluados dos modelos de medición. El primero de ello (M1) fue de tres factores oblicuos (AP, AN, y SV), y el segundo (M2) un modelo bifactor (Canivez, 2016; Reise, 2012), donde se especifica la presencia de un FG (BS) y los tres factores específicos mencionados anteriormente (AP, AN, y SV) modelados ortogonalmente.En el segundo modelo los ítems de AN fueron invertidos a fin de que todos los ítems vayan en la misma dirección y obtener un puntaje final compatible teóricamente con el BS.

El ajuste de los modelos fue evaluado mediante el RMSEA $(\leq .05)$, CFI $(\geq .95)$, SRMR $(\leq .05)$ y la prueba general $\chi^{2}$ corregida por el alejamiento de la normalidad de los ítems (SB- $\chi^{2}$; Satorra \& Bentler, 1994). Los puntos de cortes indicados para los respectivos índices fueron considerados en base a la literatura especializada (Hu \& Bentler, 1999; Byrne, 2010). Adicionalmente, para valorar el modelo bifactor fueron considerados: el coeficiente omega jerárquico general $\left(\omega_{h}\right)$ y de las subescalas $\left(\omega_{h s}\right)$ (Zinbarg et al., 2006), siendo este último sustancial cuando $\omega_{h s} \geq .30$ (Smits, Timmerman, Barelds, \& Meijer, 2015); el $H_{h}$ (>.70; Raykov, \& Hancock, 2005), que informa sobre la confiabilidad máxima de cada factor específico controlando la influencia del FG; el ECV (Sijtsma, 2009) y el PUC (Reise et al., 2013).

En cuanto a la interpretación de los dos últimos, si se observa un ECV $>.70$ y un PUC $>.70$, la varianza común puede considerarse como unidimensional (Rodriguez et al., 2016). En presencia de PUC elevados $(>.80)$, incluso ECV >.60 y $\omega_{h} \geq .70$ pueden informar favorablemente sobre la unidimensionalidad (Reise et al., 2013). Con relación a la valoración del ECV-I (Stucky et al., 2013), se espera valores $\geq .80$ (Stucky, \& Edelen, 2015). Los cálculos fueron realizados con el módulo Índices. Bifactor (Dominguez-Lara, \& Rodriguez, 2017). Cuando fue necesario, fueron consultadoslos índices de modificación de Lagrange (IM; Sörbom , 1989), a fin de detectar resultados estadísticamente significativos a nivel de ítem que ameriten un cambio en la especificación inicial (re-especificaciones) con base teórica y empírica. Por último, en cuanto al análisis de la confiabilidad, fueron calculados el coeficiente $\omega$ (McDonald, 1999) y H (Dominguez-Lara, 2016b; Hancock, \& Mueller, 2001) con el modelo que presentó evidencia más consistente. 


\section{Resultados}

\section{Análisis descriptivo}

El análisis preliminar indica que el promedio de los ítems de AP y SV está cercano a la respuesta superior, y los de AN, hacia la respuesta más baja, excepto por el ítem 15 (Nervioso) (Tabla 1). Por otro lado, los ítems poseen indicadores distribucionales (asimetría y curtosis) dentro de lo esperado (+/- 1.5; Pérez \& Medrano, 2010), e índices estandarizados de asimetría en su mayoría aceptables (SSI <.50; Malgady, 2007), lo que indicaría una dispersión tolerable.

Tabla 1

Estadísticos Descriptivos de los Ítems

\begin{tabular}{|c|c|c|c|c|c|}
\hline & $M$ & $D E$ & $g^{1}$ & SSI & $g^{2}$ \\
\hline \multicolumn{6}{|l|}{$\mathrm{AP}$} \\
\hline Item 1 & 3.359 & .892 & -.254 & .023 & .001 \\
\hline Item 3 & 2.626 & 1.183 & .250 & .165 & -.809 \\
\hline Item 5 & 3.018 & 1.047 & -.243 & .003 & -.445 \\
\hline Item 9 & 3.388 & 1.070 & -.348 & .152 & -.432 \\
\hline Item 14 & 2.964 & 1.041 & -.101 & .046 & -.509 \\
\hline Item 16 & 3.320 & 1.084 & -.309 & .131 & -.454 \\
\hline Item 17 & 3.260 & 1.059 & -.208 & .093 & -.422 \\
\hline Item 19 & 3.673 & 1.038 & -.429 & .199 & -.447 \\
\hline \multicolumn{6}{|l|}{ AN } \\
\hline Item 2 & 2.214 & 1.071 & .654 & .285 & -.352 \\
\hline Item 4 & 2.342 & 1.064 & .590 & .260 & -.275 \\
\hline Item 6 & 1.730 & 1.013 & 1.393 & .678 & 1.261 \\
\hline Item 7 & 2.107 & 1.211 & .862 & .294 & -.327 \\
\hline Item 8 & 1.961 & 1.060 & .894 & .398 & -.019 \\
\hline Item 11 & 2.488 & 1.196 & .440 & .154 & -.738 \\
\hline Item 13 & 1.641 & 0.919 & 1.441 & .852 & 1.524 \\
\hline Item 15 & 3.032 & 1.321 & -.022 & .006 & -1.155 \\
\hline Item 18 & 2.840 & 1.276 & .064 & .020 & -1.054 \\
\hline Item 20 & 2.146 & 1.200 & .853 & .296 & -.225 \\
\hline \multicolumn{6}{|l|}{ SV } \\
\hline Item 1 & 3.495 & .816 & -.022 & .017 & -.497 \\
\hline Item 2 & 3.708 & .960 & -.289 & .157 & -.639 \\
\hline Item 3 & 3.993 & .937 & -.746 & .425 & -.021 \\
\hline Item 4 & 3.459 & 1.140 & -.343 & .132 & -.685 \\
\hline Item 5 & 4.014 & .828 & -.635 & .463 & .364 \\
\hline
\end{tabular}

Nota. AP=Afecto positivo; AN=Afecto negativo; SV=Satisfacción con la vida; $M=$ media aritmética. $D E=$ desviación estándar; $g^{1}=$ asimetría de Fisher; $g^{2}=$ curtosis de Fisher. SSI=Índice estandarizado de Asimetría

\section{Evaluación de modelos de medición}

La evaluación inicial del modelo oblicuo indica que el ajuste no fue favorable $\left(\mathrm{SB}-\chi^{2}{ }_{[249]}=429.370\right.$ $[p<.01], \mathrm{CFI}=.938$, RMSEA [IC 90\%]=.051 [.043, $.059]$, SRMR =.086). Observando los IM, estos indican que se requiere una re-especificación. Concretamente, que el ítem 12 (Alerta), originalmente de AP, sea trasladado a $\mathrm{AN}\left(\chi_{[1]}^{2}=57.700 ; p<.001\right)$. Una vez realizado el cambio, el ajuste mejoró $\left(\mathrm{SB}-\chi^{2}{ }_{[249]}=408.514\right.$ $[p<.01], \quad \mathrm{CFI}=.945, \quad \mathrm{RMSEA} \quad[\mathrm{IC} 90 \%]=.048$ $[.039, .056]$, SRMR $=.077)$, aunque los IM recomiendan retornar el ítem 12 a su factor original. Ante esta situación de complejidad factorial, ya que al parecer ese ítem estaría influido simultáneamente por ambas dimensiones, se decidió eliminarlo del análisis.

Una vez retirado del modelo, el ajuste fue adecuado $\left(\mathrm{SB}-\chi^{2}{ }_{[227]}=363.900[p<.01], \mathrm{CFI}=.950, \mathrm{RMSEA}\right.$ $[\mathrm{IC} 90 \%]=.046[.037, .055], \mathrm{SRMR}=.070)$. Todas las cargas factoriales fueron estadísticamente significativas $(p<.001)$ y las correlaciones entre factores tuvieron magnitudes entre bajas y moderadas, y son compatibles con lo esperado teóricamente (p.e., correlación inversa entre AP y AN). 
El ajuste del modelo bifactor fue mejor que el oblicuo: SB- $\chi_{(207)}^{2}=308.638(p<.01), \mathrm{CFI}=.963$, RMSEA $($ IC $90 \%)=.042(.032, .051)$, SRMR $=.064$. Sin embargo, los estadísticos obtenidos indican que el FG no es lo suficientemente fuerte para ser considerado de forma independiente ya que solo explica el $15.9 \%$ de la variabilidad de las puntuaciones, mientras que los factores específicos proveen información sustancial $\left(\omega_{h s}>.30\right.$; Smits et al., 2015). A su vez, la cantidad de varianza común explicada está por debajo de lo esperado $\left(\mathrm{ECV}_{\text {total }}=.254\right.$;
PUC $=.672$ ), y adicionalmente los ítems son influidos en mayor grado por el factor específico que por el FG $\left(E C V-I_{\text {promedio }}=.285\right)$. Por tal motivo, se considera que el modelo con mejor respaldo es el de tres factores oblicuos.

Finalmente, con respecto a la confiabilidad, ambos coeficientes $\omega_{y} \mathrm{H}$ obtuvieron magnitudes aceptables en todos los casos. No fue utilizado el coeficiente alfa debido a que resulta evidente que no se cumple el principio de tau-equivalencia (Dunn, Baguley, \& Brunsden, 2014) debido a la heterogeneidad de las cargas factoriales.

Tabla 2

Parámetros de los Ítems, y Confiabilidad: Modelo Oblicuo y Bifactor

\begin{tabular}{|c|c|c|c|c|c|c|c|c|}
\hline & \multicolumn{4}{|c|}{ Modelo oblicuo } & \multicolumn{4}{|c|}{ Modelo bifactor } \\
\hline & $F_{1}$ & $\mathrm{~F}_{2}$ & $\mathrm{~F}_{3}$ & $\mathrm{~h}^{2}$ & $\mathrm{~F}_{\mathrm{E}}$ & $F_{G}$ & $\mathrm{~h}^{2}$ & ECI-I \\
\hline \multicolumn{9}{|l|}{$\mathrm{AP}$} \\
\hline Item 1 & .568 & & & .323 & .486 & .292 & .322 & .265 \\
\hline Item 3 & .337 & & & .114 & .285 & .180 & .114 & .285 \\
\hline Item 5 & .469 & & & .220 & .449 & .172 & .231 & .128 \\
\hline Item 9 & .721 & & & .520 & .612 & .398 & 533 & .297 \\
\hline Item 14 & .633 & & & .401 & .563 & .304 & .410 & .226 \\
\hline Item 16 & .622 & & & .387 & .519 & .326 & .375 & .283 \\
\hline Item 17 & .556 & & & .309 & .389 & .431 & .337 & .551 \\
\hline Item 19 & .594 & & & .353 & .453 & .375 & .346 & .407 \\
\hline \multicolumn{9}{|l|}{ AN } \\
\hline Item 2 & & .557 & & .310 & .502 & .291 & .337 & .252 \\
\hline Item 4 & & .569 & & .324 & .482 & .432 & .419 & .445 \\
\hline Item 6 & & .332 & & .110 & .276 & .252 & .140 & .455 \\
\hline Item 7 & & .654 & & .428 & .670 & .032 & .450 & .002 \\
\hline Item 8 & & .390 & & .152 & .336 & .259 & .180 & .373 \\
\hline Item 11 & & .432 & & .187 & .357 & .349 & .249 & .489 \\
\hline Item 13 & & .519 & & .269 & .479 & .203 & .271 & .152 \\
\hline Item 15 & & .747 & & .558 & .784 & .003 & .615 & .000 \\
\hline Item 18 & & .769 & & .591 & .745 & .181 & .588 & .056 \\
\hline Item 20 & & .763 & & .582 & .776 & .058 & .605 & .006 \\
\hline \multicolumn{9}{|l|}{ SV } \\
\hline Item 1 & & & .713 & .508 & .617 & .347 & .501 & .240 \\
\hline Item 2 & & & .618 & .382 & .455 & .414 & .378 & .453 \\
\hline Item 3 & & & .877 & .769 & .772 & .453 & .801 & .256 \\
\hline Item 4 & & & .578 & .334 & .470 & .327 & .328 & .326 \\
\hline Item 5 & & & .582 & .339 & .386 & .475 & .375 & .602 \\
\hline F1 & 1 & & & & & & & \\
\hline F2 & -.233 & 1 & & & & & & \\
\hline F3 & .373 & -.149 & 1 & & & & & \\
\hline$\omega$ & .790 & .835 & .810 & - & - & - & - & - \\
\hline$\omega_{h}$ & .551 & .738 & .522 & - & - & .399 & - & - \\
\hline $\mathrm{H}$ & .810 & .870 & .857 & - & - & - & - & - \\
\hline $\mathrm{H}_{h}$ & .716 & .866 & .738 & - & - & .726 & - & - \\
\hline
\end{tabular}

Nota. $F_{1}=$ Afecto positivo; $F_{2}=$ Afecto negativo; $F_{3}=$ Satisfacción con la vida; $F_{G}=$ Bienestar psicológico subjetivo; $h^{2}=$ comunalidad; ECV-I=ECV de los ítems; $\omega=$ Coeficiente omega (calculado solo para el modelo oblicuo); $\omega_{h}=$ Coeficiente omega jerárquico; $\mathrm{H}=$ coeficiente $\mathrm{H} ; \mathrm{H}_{\mathrm{h}}=$ coeficiente $\mathrm{H}$ jerárquico 


\section{Discusión}

El objetivo del presente trabajo fue evaluarla estructuralmente dos modelos explicativos del BS, uno bifactor (FG y específicos) y otro oblicuo, es decir, de tres factores específicos correlacionados.

Si bien los resultados iniciales mostraban que los índices de ajuste del modelo oblicuo no eran favorables, se observó que los IM sugerían que el ítem 12 (Alerta) al parecer estaría siendo influido tanto por el afecto negativo como por el afecto positivo. Esto implica que el ítem referido al estado de alerta connotaría tanto emoción negativa como emoción positiva. Esta ambigüedad, afecta el ajuste global del modelo por lo que se sugiere eliminarlo del análisis. Esta eliminación del ítem 12 es coincidente con otros trabajos que utilizaron la misma escala (FloresKanter \& Medrano, 2016). Una vez hecha la eliminación de dicho ítem, los índices del modelo mejoraron.

Por el otro lado, los índices de ajuste del modelo bifactor fueron superiores a los del modelo oblicuo, lo que es consistente con el trabajo de Jovanovic (2015), pero es un escenario esperado cuando compiten modelos oblicuos y jerárquicos (Gignac, 2016; Morgan et al., 2015). En este sentido el análisis conjunto de los estadísticos asociados al modelo bifactor tanto a nivel de factores $\left(\omega_{h}\right.$, ECV) como de ítems (ECI-I) indican que el FG no es lo suficientemente fuerte para ser considerado de manera independiente y, en consecuencia, para ser interpretado.

Los resultados de este trabajo indican que el modelo propuesto por Diener (1985) tiene mayor respaldo que el bifactor, lo que brinda evidencia sobre la multidimensionalidad del BS, diferenciando los aspectos afectivos y cognitivos, coincidiendo a su vez con el estudio meta-analítico revisado (Busseri, 2018). En suma, los resultados del presente trabajo apoyan la concepción multidimensional del BS.

Esta consideración diferenciada del BS puede tener implicancias a nivel teórico y clínico. En cuanto al primero, será posible orientar los estudios empíricos evaluando el componente afectivo y cognitivo de forma separada, ya que al consideradas las tres dimensiones (SV, AP, y AN) como una sola entidad, no sería posible analizar el poder explicativo de cada una de ellas sobre las variables de interés en estudios predictivos; y desde el punto de vista clínico, posibilita que los profesionales de la salud tengan en consideración la estructura multidimensional del BS al momento diseñar sus intervenciones. Por ejemplo, en un estudio se encontró que el burnout se asocia de manera inversa y significativa con la SV y con AP, mientras que dicha asociación es más fuerte con AN (Qu \& Wang, 2015). A raíz de estas diferencias, se podría pensar en una intervención para incrementar el BS planificando de forma diferenciada las estrategias para cada componente evaluado.

Además, este trabajo también aporta evidencia sobre la importancia de tener en cuenta los estadísticos complementarios al momento de analizar un modelo bifactor ya que utilizar solamente los estadísticos brindados por el análisis factorial es insuficiente para determinar el peso explicativo de cada uno de los factores específicos y, sobre todo, del FG. No obstante, lo que debe resaltarse en mayor grado es que todo modelo teórico, por simple o complejo que parezca, debe ser sometido a prueba.

Con todo, el presente trabajo presenta algunas limitaciones que deben ser consideradas para la realización de futuros trabajos. Con respecto a la muestra seleccionada vale destacar que, si bien conserva las condiciones necesarias para realizar un AFC, podría resultar interesante contar con una muestra mayor con fines de replicación. También se debe tener en cuenta que la población utilizada para este trabajo consistió en jóvenes universitarios, por lo que los resultados no pueden ser generalizados a otras poblaciones. Por tal motivo, sería necesaria una réplica del estudio con un mayor tamaño muestral y bajo un enfoque de invarianza de medición considerando un análisis diferenciado por sexo. Del mismo modo, sería conveniente un estudio en un contexto cultural distinto debido a que la experiencia de BS se encuentra mediado por las vivencias del individuo en su entorno ( $\mathrm{Lu}, 2008)$. El modelamiento bifactor no está exento de críticas (Eid, Geiser, Koch, \& Heene, 2016), por lo que sus resultados deben ser interpretados con precaución (Bonifay, Lane, $\&$ Reise, 2017). Finalmente, si bien se obtuvo evidencia de validez de constructo respecto al modelo oblicuo, futuras investigaciones podrían hacer hincapié también en la validez predictiva de este modelo proponiendo relaciones con variables externas (Tornimbeni, Pérez, \& Olaz, 2008).

Para concluir, los hallazgos de este estudio sugieren quelas variaciones de las puntuaciones son mejor explicadas por el modelo oblicuo con los tres factores correlacionados. Sin embargo, para poder explicar mejor la estructura del BS sería apropiado conducir más investigaciones que aborden este tema.

\section{Referencias}

Bentler, P. M., \& Wu, E. J. C. (2012). EQS 6.2 for windows [Statistical Program]. Encino, CA: Multivariate Software, Inc.

Bonifay, W., Lane, S. P., \& Reise, S. P. (2017). Three concerns with applying a bifactor model as a structure of psychopathology. Clinical Psychological Science, 5(1), 184-186. doi: 10.1177/2167702616657069 
Busseri M. A. (2015). Toward a Resolution of the tripartite structure of subjective well-being. Journal of Personality, 83(4),413-428. doi: 10.1111/jopy.12116

Busseri, M. A. (2018). Examining the structure of subjective well-being through meta-analysis of the associations among positive affect, negative affect, and life satisfaction. Personality and Individual Differences, 122(2), 68-71. doi: 10.1016/j.paid.2017.10.003

Busseri M. A., \& Sadava S. W. (2011) A review of the tripartite structure of subjective well-being: Implications for conceptualization, operationalization, analysis, and synthesis. Personality and Social Psychology Review, 15(3), 290-314. doi:10.1177/1088868310391271

Byrne, B. M. (2010). Structural equation modeling with AMOS. Basic concepts, applications, and programming. New York: Routledge.

Canivez, G. L. (2016). Bifactor modeling in construct validation of multifactored tests: Implications for multidimensionality and test interpretation. Em K. Schweizer \& C. DiStefano (Eds.), Principles and methods of test construction: Standards and recent advancements (pp. 247-271). Gottingen, Germany: Hogrefe.

Diener E., Lucas R. E., \& Oishi S. (2002). Handbook of positive psychology. New York: Oxford University Press.

Diener, E. (1985). The satisfaction with life scale. Journal of Personality Assessment. 49(1), 71-75. doi:10.1207/s1532775ipa4901_13

Dominguez-Lara, S. (2014). ¿Matrices Policóricas/Tetracóricas o Matrices Pearson? Un estudio metodológico. Revista Argentina de Ciencias del Comportamiento, 6(1), 39-48. Recuperado de http://www.redalyc.org/articulo.oa?id=333430869006

Dominguez-Lara, S. (2016a). Evaluación de modelos estructurales, más allá de los índices de ajuste. Enfermería Intensiva, 27(2), 84-85. doi: 10.1016/j.enfi.2016.03.003

Dominguez-Lara, S. (2016b). Evaluación de la confiabilidad del constructo mediante el Coeficiente H: Breve revisión conceptual y aplicaciones. Psychologia: Avances en la disciplina, 10(2), 87-94. doi: 10.21500/19002386.2134

Dominguez-Lara, S., \& Rodriguez, A. (2017). Índices estadísticos de modelos bifactor. Interacciones, 3(2), 59-65. doi: 10.24016/2017.v3n2.51

Dunn, T. J., Baguley, T., \& Brunsden, V. (2014). From alpha to omega: A practical solution to the pervasive problem of internal consistency estimation. British Journal of Psychology, 105(3), 399-412. doi: 10.1111/bjop.12046

Eid, M., Geiser, C., Koch, T., \& Heene, M. (2016). Anomalous results in g-factor models: Explanations and alternatives. Psychological Methods, 22(3), 541-562. doi: 10.1037/met0000083

Flores-Kanter, P. E., \& Medrano L.A. (2016). El afecto y sus dimensiones: Contrastes de modelos ortogonales y oblicuos mediante análisis factorial confirmatorio de la escala PANAS. Liberabit, 22(2), 173-184. Recuperado de http://www.redalyc.org/articulo. oa?id $=68649318004$

García-Viniegras, C. R. V., \& González-Benítez, I. (2000). La categoría bienestar psicológico: Su relación con otras categorías sociales. Revista Cubana de Medicina General Integral, 16(6), 586-592. Recuperado de http://scielo.sld.cu/scielo.php?script=sci_arttext\&pid $=$ S0864-21252000000600010

Gignac, G. E. (2016). The higher-order model imposes a proportionality constraint: That is why the bifactor model tends to fit better. Intelligence, 55, 57-68. . doi: 10.1016/j.intell.2016.01.006

Hancock, G. R., \& Mueller, R. O. (2001). Rethinking construct reliability within latent variable systems. Em R. Cudeck, S. H. C. du Toit \& D. Sörbom (Eds.), Structural equation modeling: Past and present. A Festschrift in honor of Karl G. Jöreskog (pp. 195-261). Chicago: Scientific Software International.

Hu, L. \& Bentler, P. M. (1999). Cutoff criteria for fit indexes in covariance structure analysis: Conventional criteria versus new alternatives. Structural Equation Modeling: A Multidisciplinary Journal, 6(1), 1-55. doi: 10.1080/10705519909540118

Jovanović, V. (2015). A bifactor model of subjective well-being: A re-examination of the structure of subjective well-being. Personality and Individual Differences, 87, 45-49. doi: 10.1016/j.paid.2015.07.026

Lee, S. Y., Poon, W. Y., \& Bentler, P. M. (1995). A two-stage estimation of structural equation models with continuous and polytomous variables. British Journal of Mathematical and Statistical Psychology, 48(2), 339-358. doi: 10.1111/j.2044-8317.1995.tb01067.x

Lu, L. (2008). Culture, self, and subjective well-being: Cultural psychological and social change perspectives. Psychologia, 51(4), $290-303$. doi: $10.2117 /$ psysoc. 2008.290

Luhmann M. (2017). The development of subjective well-being. In J: Specht, Personality Development Across the Lifespan (pp. 197-218). Cambridge, Massachusetts: Academic Press.

Malgady, R. (2007). How skew are psychological data? A standardized index of effect size. The Journal of General Psychology, 134(3), 355-359. doi: 10.3200/GENP.134.3.355-360

McDonald R. P. (1999). Test theory: A unified treatment. Mahwah, N. J.: L. Erlbaum Associates.

Medrano, L.A., Flores-Kanter, P.E., Trógolo M., Curarello A., \& González J. (2015). Adaptación de la Escala de Afecto Positivo y Negativo (PANAS) para la población de Estudiantes Universitarios de Córdoba. Anuario de investigaciones de la facultad de psicología, 2(1), 22-36. Recuperado de https://revistas.unc.edu.ar/index.php/aifp/article/download/12503/12773.

Morgan, G. B., Hodge, K. J., Wells, K. E., \& Watkins, M. W. (2015). Are fit indices biased in favor of bi-factor models in cognitive ability research? A comparison of fit in correlated factors, higher-order, and bi-factor models via Monte Carlo simulations. Journal of Intelligence, 3(1), 2-20. doi: 10.3390/jintelligence3010002

Moyano, N. C., Martínez Tais, M., \& Muñoz, M. P. (2013). Propiedades Psicométricas de la Escala de Satisfacción con la Vida de Diener. Revista Argentina de Clínica Psicológica, 12(2), 161-168. Recuperado de http://www.redalyc.org/html/2819/281931436008/

Olivier P. R., Navarro-Guzmán J. I., Menacho-Giménez, I., López-Sinoga, M. M., \& García-Sedeño, M. A. (2016). Bienestar psicológico en personas con alta capacidad intelectual. European Journal of Education and Psychology, 9(2), 72-78. doi: 10.1016/j.ejeps.2015.12.001

Oliveira, C., Nunes, M. F. O., Legal, E. J., \& Noronha, A. P. P. (2016). Bem-estar subjetivo: Estudo de correlação com as forças de caráter. Avaliação Psicológica, 15(2), 177-185. Recuperado de http://pepsic.bvsalud.org/scielo.php?script=sci_arttext\&pid=S1677-04712016000200007

Pérez, E. \& Medrano, L. (2010). Análisis factorial exploratorio: Bases conceptuales y metodológicas. Revista Argentina de Ciencias del Comportamiento, 2(1), 58-66. Recuperado de http://www.redalyc.org/html/3334/333427068006/

Qu, H. Y., \& Wang C. M. (2015) Study on the relationships between nurses' job burnout and subjective well-being. Chinese Nursing Research, 2(2/3), 61-69. doi: 10.1016/j.cnre.2015.09.003

Raykov, T., \& Hancock, G. R. (2005). Examining change in maximal reliability for multiple-component measuring instruments. British Journal of Mathematical and Statistical Psychology, 58(1), 65-82. doi: 10.1348/000711005X38753

Reise, S. P. (2012). The rediscovery of bifactor measurement models. Multivariate Behavioral Research, 47(5), $667-696$. doi: $10.1080 / 00273171.2012 .715555$ 
Reise, S. P. Scheines, R., Widaman, K. F., \& Haviland, M. G. (2013). Multidimensionality and structural coefficient bias in structural equation modeling: A bifactor perspective. Educational and Psychological Measurement, 73(1), 5-26. doi: 10.1177/0013164412449831

Rodriguez, A., Reise, S. P., \& Haviland, M. G. (2016). Evaluating bifactor models: Calculating and interpreting statistical indices. Psychological Methods, 21(2), 137-150. doi: 10.1037/met0000045

Satorra, A., \& Bentler, P. M. (1994). Corrections to test statistics and standard errors in covariance structure analysis. Em A. von Eye \& C. C. Clogg (Eds.), Latent variables analysis: Applications for developmental research (pp. 399-419). Thousand Oaks, CA: Sage.

Sijtsma, K. (2009). On the use, the misuse, and the very limited usefulness of Cronbach's alpha. Psychometrika, 74(1), 107-120. doi: 10.1007/ s11336-008-9101-0

Smits, I. A. M., Timmerman, M. E., Barelds, D. P. H., \& Meijer, R. R. (2015). The Dutch symptom checklist-90-revised: Is the use of the subscales justified? European Journal of Psychological Assessment, 31(4), 263-271. doi: 10.1027/1015-5759/a000233

Sörbom, D. (1989). Model modification. Psychometrika, 54(3), 371-384. doi: 10.1007/BF02294623

Stucky, B. D., \& Edelen, M. O. (2015). Using heierarchical IRT models to create unidimensional measures from multidimensional data. Em S. P. Reise \& D. A. Revicki (Eds.), Handbook of item response theory modeling: Applications to typical performance assessment (pp. 183-206). New York: Routledge.

Stucky, B. D., Thissen, D., \& Edelen, M. O. (2013). Using logistic approximations of marginal trace lines to develop short assessments. Applied Psychological Measurement, 37(1), 41-57. doi: 10.1177/0146621612462759

Tornimbeni, S., Pérez, E. \& Olaz, F. (2008). Introducción a la Psicometría. Córdoba, Argentina: Brujas.

Watson, D., Clark, L. A., \& Tellegen, A. (1988). Development and validation of brief measures of positive and negative affect: the PANAS scales. Journal of Personality and Social Psychology, 54(6), 1063-1070. doi: 10.1037/0022-3514.54.6.1063

Zinbarg, R. E., Yovel, I., Revelle, W., \& McDonald, R. P. (2006). Estimating generalizability to a latent variable common to all of a scale's indicators: A comparison of estimators for $\omega_{h}$. Applied Psychological Measurement, 30(2), 121-144. doi: 10.1177/0146621605278814

\section{Sobre os autores}

Lucas Lapuente es Psicólogo. Estudiante de posgrado. Investigador part-time en la Universidad Siglo 21 (Argentina).

Sergio Dominguez-Lara es Psicólogo, y Magíster en Psicología Clínica y de la Salud. Docente del Instituto de Investigación de Psicología (USMP, Perú).

Pablo Ezequiel Flores-Kanter es Psicólogo. Estudiante de posgrado. Investigador part-time en la Universidad Siglo 21 (Argentina).

Leonardo Adrián Medrano es Psicólogo y Doctor en Psicología. Secretario de Investigación en la Universidad Siglo 21 (Argentina). 